ARTICLE

https://doi.org/10.1038/s41467-021-23436-x

\title{
High-responsivity graphene photodetectors integrated on silicon microring resonators
}

S. Schuler ${ }^{1,2,7}$, J. E. Muench ${ }^{2,7}$, A. Ruocco ${ }^{2}$, O. Balci ${ }^{2}$, D. van Thourhout (10 ${ }^{3}$, V. Sorianello (10 ${ }^{4}$, M. Romagnoli ${ }^{4}$,

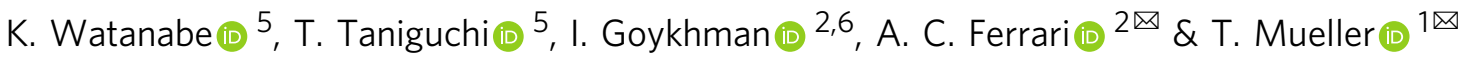

Graphene integrated photonics provides several advantages over conventional Si photonics. Single layer graphene (SLG) enables fast, broadband, and energy-efficient electro-optic modulators, optical switches and photodetectors (GPDs), and is compatible with any optical waveguide. The last major barrier to SLG-based optical receivers lies in the current GPDs' low responsivity when compared to conventional PDs. Here we overcome this by integrating a photo-thermoelectric GPD with a Si microring resonator. Under critical coupling, we achieve $>90 \%$ light absorption in a $6 \mu \mathrm{m} \mathrm{SLG} \mathrm{channel} \mathrm{along} \mathrm{a} \mathrm{Si} \mathrm{waveguide.} \mathrm{Cavity-enhanced} \mathrm{light-}$ matter interactions cause carriers in SLG to reach $\sim 400 \mathrm{~K}$ for an input power $\sim 0.6 \mathrm{~mW}$, resulting in a voltage responsivity $\sim 90 \mathrm{~V} / \mathrm{W}$, with a receiver sensitivity enabling our GPDs to operate at a $10^{-9}$ bit-error rate, on par with mature semiconductor technology, but with a natural generation of a voltage, rather than a current, thus removing the need for transimpedance amplification, with a reduction of energy-per-bit, cost, and foot-print.

\footnotetext{
${ }^{1}$ Vienna University of Technology, Institute of Photonics, Vienna, Austria. ${ }^{2}$ Cambridge Graphene Centre, University of Cambridge, Cambridge, UK. ${ }^{3}$ Ghent University-IMEC, Photonics Research Group, Gent, Belgium. ${ }^{4}$ Consorzio Nazionale per le Telecomunicazioni and Inphotec, Pisa, Italy. ${ }^{5}$ National Institute for Materials Science, Tsukuba, Japan. ${ }^{6}$ Technion-Israel Institute of Technology, Haifa, Israel. ${ }^{7}$ These authors contributed equally: S. Schuler, J. E. Muench.

凶email: acf26@eng.cam.ac.uk; thomas.mueller@tuwien.ac.at
} 
T he same-chip integration ${ }^{1}$ of active and passive optical components with electronics offers a cost- and energy-efficient solution for short- and long-reach optical interconnects ${ }^{2,3}$. Single layer graphene (SLG) is an ideal material for integrated photonics ${ }^{4,5}$, promising e.g. high-speed $(>200 \mathrm{GHz})^{6,7}$ and broadband (ultraviolet to far-infrared) $)^{8,9}$ operation that could lift bandwidth (BW) $(\sim 100 \mathrm{GHz})^{10,11}$ and spectral $(<1600 \mathrm{~nm})^{12}$ limitations of existing technologies, such as $\mathrm{Ge} / \mathrm{Si}^{13,14}$ and $\mathrm{InGaAsP} / \mathrm{InP}^{15,16}$. A variety of waveguide (WG)-integrated SLG-based photonic devices have been reported ${ }^{17-34}$, including electro-absorption (EAMs) ${ }^{17-19}$ and electro-refraction modulators (ERMs) ${ }^{20}$, optical switches ${ }^{4,21}$, and photodetectors (GPDs) ${ }^{22-33}$. SLG and layered materials can be integrated with passive Si photonic $\mathrm{WGs}^{22-27}$ or any other passive WG technology ${ }^{4}$, including $\mathrm{Si}_{3} \mathrm{~N}_{4}{ }^{29,34,35}$, sapphire ${ }^{36}, \mathrm{Ge}^{37}$, and polymers $^{38,39}$, extending the spectral range and scope of possible applications 37,40 .

SLG's optical absorption is $\sim 2.3 \%$ under normal incidence ${ }^{41}$, which limits the photoresponse in top-illuminated GPDs ${ }^{8}$. This can be increased in a WG configuration through the interaction with the evanescent field of the optical WG mode ${ }^{42}$. However, since the mode-overlap with SLG's monatomic cross-section typically restricts absorption to $\sim 0.01-0.1 \mathrm{~dB} / \mu \mathrm{m}(\sim 0.2-2 \% / \mu \mathrm{m})^{4}$, device lengths $\sim 100 \mu \mathrm{m}$ are needed for near-complete $(>90 \%)$ absorption, with adverse effects on foot-print and capacitance ${ }^{7}$. The resulting trade-off between length and absorption has implications for GPDs that operate via the photo-thermoelectric effect $(\mathrm{PTE})^{43-45}$ : Due to slow $\left(\sim \mathrm{ps}^{46}\right)$ heat dissipation to the lattice via phonon mediated cooling 46,47 , photo-excitation leads to the formation of a hot-carrier distribution in $\mathrm{SLG}^{8,43,44}$. The associated electron temperature, $T_{e}$, can be substantially above the lattice temperature, $T_{0}{ }^{48}$, and leads to a photovoltage ${ }^{43-45}$ :

$$
V_{\mathrm{PTE}}=\int S(x) \cdot \nabla T_{e}(x) \mathrm{d} x
$$

if both a $T_{e}$ gradient and a spatially varying Seebeck coefficient, $S$, (controlled by the chemical potential, $\mu_{c}$ ) are present ${ }^{43}$. In order to achieve a high $(>\mathrm{mV}) V_{\mathrm{PTE}}$, it is better to absorb the incident electromagnetic energy over small $(<10 \mu \mathrm{m})$ lengths, leading to localised electronic heating for a higher ( tens $\mathrm{K}) T_{e}$ and $\nabla T_{e}(x)$.

Different approaches have been explored to increase and confine light absorption in free-space-coupled ${ }^{49-51}$ and WGintegrated GPDs ${ }^{26-29}$, e.g. by embedding SLG into optical cavities $^{50,51}$, slot $\mathrm{WGs}^{26}$, plasmonic structures ${ }^{28,29,31,49}$, or by enhancing the light-matter interaction using sub-wavelength structures $^{27}$, but these have coupling and propagation losses ${ }^{31}$, limitations in field enhancement ${ }^{25,30}$, or in carrier mobility $\mu^{26,32}$, fabrication flows incompatible with complementary metal-oxidesemiconductor (CMOS) processing $25,31,32$, bias-induced dark currents $^{30,31}$, or a combination thereof ${ }^{25,31,32}$. Thus, the demonstration of GPDs on photonic integrated circuits (PICs) that leverage SLG's unique hot-carrier dynamics and maximise the voltage responsivity $R_{[\mathrm{V} / \mathrm{W}]}=V_{\mathrm{PTE}} / P_{\mathrm{in}}$, with $P_{\text {in }}$ the incident optical power in the $W G$, is challenging.

Here, we report GPDs integrated on looped WGs, known as microring resonators ${ }^{52}$, which act as PIC-embedded resonant cavities. The conversion of incident light into an electrical signal occurs via the PTE effect. The GPDs directly generate a voltage, which allows us to operate them without bias and dark currents, limiting the GPD noise to thermal Johnson-noise, due to fluctuations of the carrier density ${ }^{53}$. Other sources of noise, in particular low $(\sim \mathrm{Hz}-\mathrm{kHz})$ frequency contributions, such as $1 / \mathrm{f}$ and generation-recombination ${ }^{54}$, are not relevant for bias-free high-speed PTE-GPDs operating in the $\mathrm{GHz}$ regime ${ }^{33}$. This removes the need of transimpedance amplifiers (TIA) in the read-out electronics, with a reduction of the energy-perbit cost and system foot-print. With $R_{[\mathrm{V} / \mathrm{W}]} \sim 90 \mathrm{~V} / \mathrm{W}$, our GPDs pave the way towards SLG integration on $\mathrm{Si}$ photonic receivers, overcoming the limitation of photocurrent $\left(I_{\mathrm{ph}}\right)$ generating GPDs with current responsivities $R_{[\mathrm{A} / \mathrm{W}]}=I_{\mathrm{ph}} / P_{\text {in }}$ lower than mature Ge $\mathrm{PDs}^{25,30,31}$. We attribute this to our high $\left(>10^{4} \mathrm{~cm}^{2} / \mathrm{Vs}\right) \mu$, and the combination with the $\mathrm{Si}$ microring resonator giving a $\sim 10$-fold enhancement of electric field strength and $>90 \%$ light absorption in just $\sim 6 \mu \mathrm{m}$ SLG on Si WGs.

\section{Results}

Device concept. Figure 1 a is a scheme of our GPDs, which comprise a layered materials heterostructure (LHM) of SLG and hexagonal boron nitride $(\mathrm{hBN})$. To increase the generated $V_{\mathrm{PTE}}$ upon optical illumination according to Eq. (1), encapsulation of the SLG channel in hBN ensures a high $\left(>10^{4} \mathrm{~cm}^{2} / \mathrm{Vs}\right) \mu^{55}$ for large $(\sim 200 \mu \mathrm{V} / \mathrm{K})$ peak $S^{29}$, according to Mott's formula ${ }^{43,44}$ :

$$
S=-\frac{\pi^{2} k_{\mathrm{B}}^{2} T_{e}}{3 e \sigma\left(\mu, \mu_{c}\right)} \frac{\mathrm{d} \sigma\left(\mu, \mu_{c}\right)}{\mathrm{d} \mu_{c}}
$$

where $k_{\mathrm{B}}$ is the Boltzmann constant, $e$ the electron charge, $\sigma=n \mu e$ the conductivity, and $n$ the carrier concentration. Dual-gate SLG electrodes, separated from the $\mathrm{LMH}$ by an $\mathrm{Al}_{2} \mathrm{O}_{3}$ layer, are employed to tune $S$ in adjacent regions of the device ${ }^{26}$. The $\mathrm{LMH}$ is contacted on opposite sides and centrally aligned to the WG of a microring resonator fabricated on a Si-on-insulator (SOI) wafer. The resonator serves a two-fold purpose. First, the higher (compared to the bus WG) intra-cavity energy density ${ }^{52}$ results in $\sim 10$-fold enhanced lightmatter interaction ${ }^{56}$ and can enable near-complete light absorption in the SLG channel if its coverage of the resonator is optimised. Second, the wavelength, $\lambda$, selectivity of the resonator ${ }^{56,57}$, makes the GPD suitable for wavelength division multiplexing $(\mathrm{WDM})^{14}$, whereby the data rate of a single optical channel is increased by combining signals of different $\lambda$ at the transmitter, and separating them at the receiver ${ }^{58-60}$.

To find the SLG length, $W$, over the ring that enables maximum absorption inside the resonator, we perform an initial experiment on a reference microring cavity with identical parameters (WG thickness $t_{\mathrm{WG}}=220 \mathrm{~nm}$, WG width $w_{\mathrm{WG}}=480 \mathrm{~nm}$, ring radius $R=40 \mu \mathrm{m})$. Coupling to the resonator occurs through a $200 \mathrm{~nm}$ gap via a single bus WG, Fig. 1a, which has grating couplers (GCs) on either end, with coupling efficiency ${ }^{14} \eta=P_{\text {in }} / P_{\text {fibre }} \sim 0.28$, with $P_{\text {fibre }}$ the optical power in the fibre connecting source and SOI chip, determined from transmission measurements on reference WGs on the same chip. The power coupling between these two structures depends on the coupling and transmission coefficients, i.e. the scattering matrix elements relating incoming and outgoing electric fields from the coupling region ${ }^{56,57}$. The choice of the power coupling coefficient, $\kappa$, affects both the optical (frequency) bandwidth $f_{\mathrm{FWHM}}$ and the quality factor $\mathrm{Q}=f_{\mathrm{FWHM}} / f_{\text {res }}$, with $f_{\text {res }}$ the resonance frequency ${ }^{59}$ of the resonator, resulting in a trade-off between achievable extinction ratio (ER, defined as the ratio of minimum (at resonance) and maximum transmitted optical power ${ }^{50}$ ), thus $R_{[\mathrm{V} / \mathrm{W}]}$, and the maximum achievable electrical bandwidth, $f_{3 \mathrm{~dB}}$. For our GPD, we select $\kappa=10 \%$ (with a measured optical (wavelength) bandwidth $\lambda_{\text {FWHM }} \sim 150 \mathrm{pm}$ at $\lambda_{\text {res }} \sim 1.55 \mu \mathrm{m}$, corresponding to $f_{\mathrm{FWHM}} \sim 18.7 \mathrm{GHz}$. This, according to ${ }^{61}$ :

$$
f_{3 \mathrm{~dB}}=\sqrt{\sqrt{2}-1} \times f_{\mathrm{FWHM}}
$$

allows for $f_{3 \mathrm{~dB}} \sim 12 \mathrm{GHz}$ in our design, sufficient for applications in data centre optical interconnects ${ }^{2}$.

The wavelength-dependent transmitted power, $P_{\text {trans }}$, of a ring resonator can be written as ${ }^{52}$ :

$$
P_{\text {trans }}=P_{\text {in }} \frac{(1-\kappa)+\xi-2 \sqrt{(1-\kappa) \xi} \cos (\theta)}{1+(1-\kappa) \xi-2 \sqrt{(1-\kappa) \xi} \cos (\theta)}
$$

where $\theta=4 \pi^{2} R n_{\text {eff }} / \lambda$ is the round-trip phase shift of the 
a

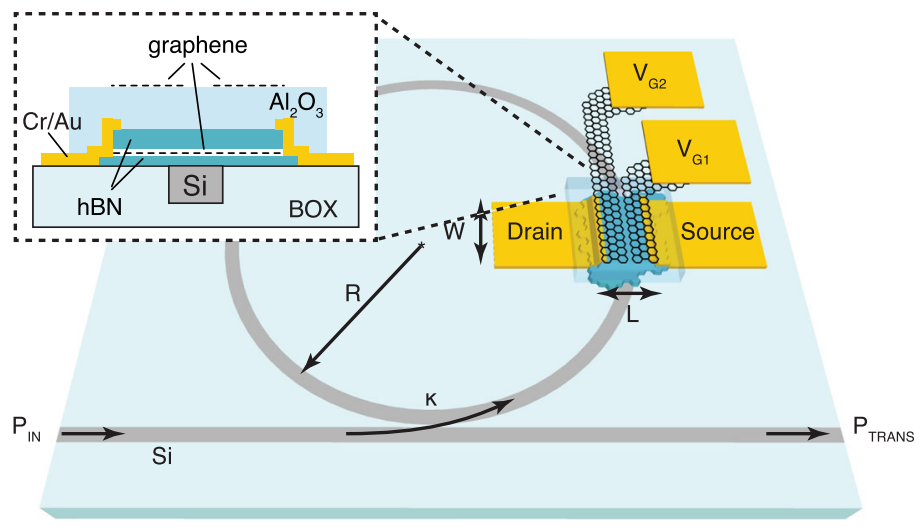

b

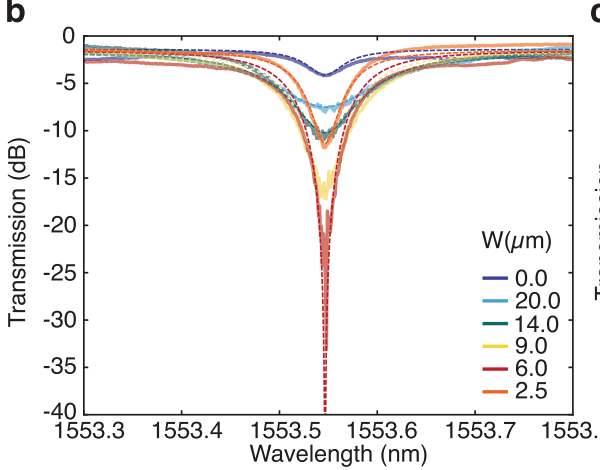

C

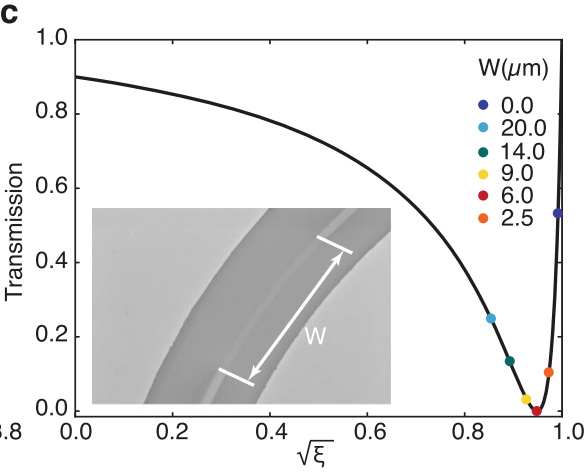

Fig. 1 Si ring resonator integrated GPD. a Sketch of device. $\mathbf{b}$ Transmission spectra of a ring cavity with SLG on top, indicating the effects of varying $W$ on ER and Q. For clarity, the spectra are aligned at the resonance wavelength closest to $1553.55 \mathrm{~nm}$. c Corresponding transmission at resonance. The black line is the calculated transmission for $\kappa=10 \%$. The coloured dots mark the transmission for various loss coefficients.

circulating mode and the effective mode index $n_{\text {eff }}=\beta_{m} / k_{0}$, with $\beta_{m}$ the propagation constant of the mode, defined as the wavevector component along the WG, and $k_{0}$ the free-space wavevector ${ }^{60,62}$. Omitting negligible losses caused by coupling between bus and ring, the term:

$$
\xi=e^{-W \alpha_{\mathrm{SLG}}} e^{-2 \pi R \alpha_{\mathrm{WG}}}
$$

describes the round-trip propagation loss in the ring, with $\alpha_{\mathrm{SLG}}$ and $\alpha_{\mathrm{WG}}$, in $\mathrm{dB} / \mu \mathrm{m}$, the power attenuation coefficients in SLG and $\mathrm{Si}$ WG, respectively. When $\theta=2 \pi m(m=1,2,3 \ldots)$, the light in the ring constructively interferes with itself and the cavity is in resonance ${ }^{52}$. From Eq. (4), the transmission drops to zero if $\xi=1-\kappa$. Under this so-called critical coupling ${ }^{57}$, as the transmission approaches zero, maximum absorption inside the ring resonator is achieved. With all other parameters fixed in Eq. (5), changing the SLG-induced losses by changing $W$ can therefore be used to tune $\xi$ and achieve critical coupling.

Coupling and absorption optimisation. To find the optimum $W$, we first measure the transmission of an unloaded (no SLG, i.e. $W=0 \mu \mathrm{m}$ ) resonator by coupling light (continuous-wave (CW), TE-polarised) from a tunable laser (Newport TLB6700) into the bus WG, using an optical single-mode fibre, and measuring the transmitted power at the output GC as a function of $\lambda$, around one of the resonance peaks close to main Telecom wavelength at $1.55 \mu \mathrm{m}$, Fig. 1b. Due to SLG's broadband absorption ${ }^{8}$, there will be identical behaviour for the other resonances, apart from a shift in $P_{\text {in }}$ with GC response envelope ${ }^{14}$. The results, after calibration for the coupling losses, are shown by the dark-blue line and symbols in Figs. 1b, c, respectively. The microring resonator is not critically coupled at resonance for $\lambda \sim 1553.55 \mu \mathrm{m}$, as $P_{\text {trans }}$ does not vanish, but only part of the incident power is dissipated in the WG. From Eqs. (4), (5), $\alpha_{\mathrm{WG}} \sim 1.4 \mathrm{~dB} / \mathrm{cm}$.

We then proceed to study the effect of SLG with varying $W$ on the power dissipated in the resonator. We first place a $W=20 \mu \mathrm{m}$ SLG flake, prepared by micro-mechanical cleavage $(\mathrm{MC})^{63}$ of bulk graphite, transferred using a micro-manipulator and a stamp consisting of polycarbonate (PC) and polydimethylsiloxane (PDMS), and cleaned by immersion in chloroform, over the ring, and measure the transmission as before. Using successive electron beam lithography (EBL, Raith e-LINE) runs to define a poly(methyl methacrylate) (PMMA) etch mask and reactive ion etching in $\mathrm{O}_{2}$ to remove excess material, we then reduce $W$ further in several steps down to $2.5 \mu \mathrm{m}$, with transmission measurements in between. The results, Figs. 1b, c, show an initial transmission decrease at resonance with decreasing $W$, before the trend is inverted as $W$ tends to zero. The minimum transmission, indicating critical coupling, is for $W=$ $6 \mu \mathrm{m}$. From Eq. (5) we extract $\alpha_{\mathrm{G}} \sim 0.07 \mathrm{~dB} / \mu \mathrm{m}$, in agreement with measured ${ }^{17}$ and simulated ${ }^{4}$ values from literature. Using these and the comparison of the transmission curves for $W=6 \mu \mathrm{m}$, we estimate the fraction of absorbed light in the SLG channel to be $~ 92 \%$ under critical coupling. Figure $1 \mathrm{~b}$ can also be used to monitor the effects of changing $W$ on $Q$, as discussed in Supplementary Note 1. Supplementary Fig. 1 plots the degradation of $Q$ as losses are increased with $W$. We further use $Q^{56,64}$ to confirm the absorption in our devices.

LMH characterisation. Based on these findings, we fabricate the GPD in Fig. 1a with $W=6 \mu \mathrm{m}$ from a LMH (hBN encapsulated SLG as channel layer) on top of the ring resonator, as shown in 
a

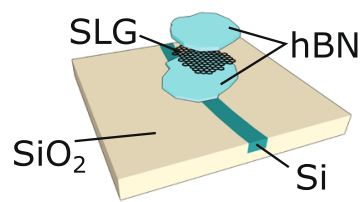

d

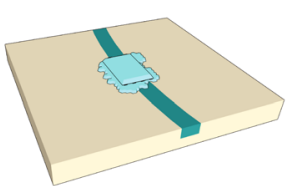

g

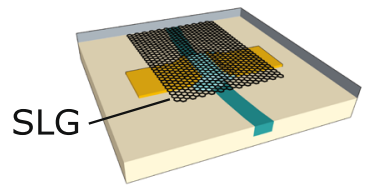

b

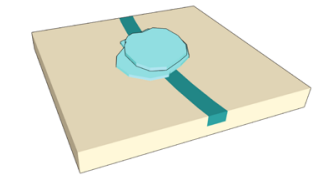

C



e
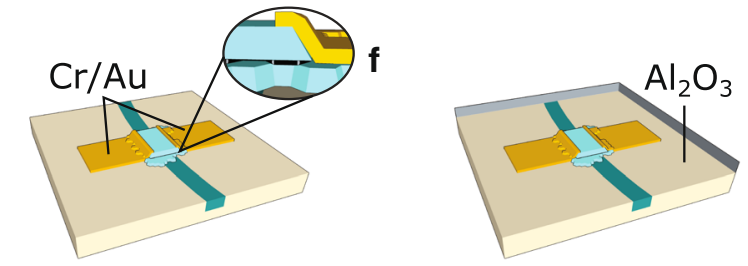

h

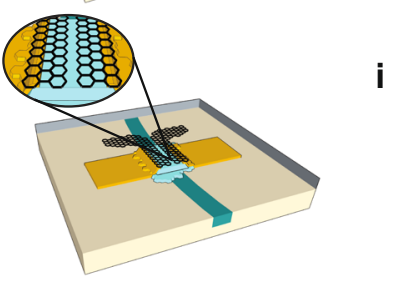

i



Fig. 2 Device fabrication. a Assembly of $h B N / S L G / h B N$. b Stack placement on photonic circuit and interface cleaning. $\mathbf{c} h B N$ etching in $S F_{6}$ plasma. $\mathbf{d} S L G$ etching in $\mathrm{O}_{2}$ plasma to define channel geometry. e Metallization $(\mathrm{Cr} / \mathrm{Au})$ for drain-source contacts. $\mathbf{f} \mathrm{Al}$ seed layer evaporation and $\mathrm{ALD}$ of $\mathrm{Al}_{2} \mathrm{O}_{3}$. $\mathbf{g}$ Wet transfer of CVD SLG. h Split-gate fabrication. i Metallization ( $\mathrm{Cr} / \mathrm{Au}$ ) for gate contacts.

Fig. 2 and described in Methods. A microscope image of hBN/ SLG/hBN on the WG is in Fig. 3a. We perform Raman spectroscopy (Renishaw inVia at $514.5 \mathrm{~nm}$, power $<0.5 \mathrm{~mW}$ ) and atomic force microscopy (AFM, Bruker Dimension Icon) to monitor the SLG quality. A typical Raman spectrum before further processing of the stack is in Fig. 3b. The position of the combined hBN $\mathrm{E}_{2 \mathrm{~g}}$ peaks ${ }^{65}$ from top and bottom flakes is Pos $\left(E_{2 \mathrm{~g}}\right) \sim 1366 \mathrm{~cm}^{-1}$ with full-width half maximum, $\operatorname{FWHM}\left(E_{2 \mathrm{~g}}\right) \sim$ $9.5 \mathrm{~cm}^{-1}$, as expected considering the top flake is bulk and that the planar domain size in MC-produced hBN crystals is limited by the flake size ${ }^{55,66}$. Pos(2D) $2693 \mathrm{~cm}^{-1}, \mathrm{FWHM}(2 \mathrm{D})$ $\sim 18 \mathrm{~cm}^{-1}, \quad \operatorname{Pos}(\mathrm{G}) \sim 1583 \mathrm{~cm}^{-1}, \quad \operatorname{FWHM}(\mathrm{G}) \sim 14 \mathrm{~cm}^{-1}$, confirming the presence of SLG and low $n<10^{12} \mathrm{~cm}^{-267}$. The area $(\mathrm{A}(2 \mathrm{D}) / \mathrm{A}(\mathrm{G}) \sim 10.7)$ and intensity $(\mathrm{I}(2 \mathrm{D}) / \mathrm{I}(\mathrm{G}) \sim 7.6)$ ratios indicate a Fermi level $E_{\mathrm{F}}<100 \mathrm{meV}^{67-69}$.

The AFM scan of the overlap region between $\mathrm{LMH}$ and microring in Fig. $3 \mathrm{c}$ shows blister-free $\mathrm{SLG} / \mathrm{hBN}$ interfaces, confirming successful cleaning ${ }^{55}$, apart from a bubble trapped in a cladding trench above the WG. The FWHM(2D) map in Fig. 3d, taken from a $20 \times 30 \mu \mathrm{m}^{2}$ area in the centre of the $\mathrm{LMH}$, shows a region with homogeneous (spread $\left.<1 \mathrm{~cm}^{-1}\right)$ and narrow $(\leq 18 \mathrm{~cm}$ $\left.{ }^{-1}\right) \operatorname{FWHM}(2 \mathrm{D})$ and spots of increased $\left(>21 \mathrm{~cm}^{-1}\right) \operatorname{FWHM}(2 \mathrm{D})$ that coincide with the blister position, as revealed by AFM. Based on these findings, we then select the channel position (marked red in Figs. 3a, c, d) to be in a blister-free region. The final device has an active area $L \times W \sim 2.5 \times 6 \mu \mathrm{m}^{2}$. We use $L \sim 2.5 \mu \mathrm{m}$, of the order of twice the cooling length $L_{\text {cooling }}$ in SLG $\left(\sim 1 \mu \mathrm{m}^{25,70}\right.$, related to electron thermal conductivity $\kappa_{e}$ (see Methods) and interfacial heat conductivity $\Gamma \sim 0.5-5 \mathrm{MWm}^{-2} \mathrm{~K}^{-171,72}$, via $L_{\text {cooling }}=\sqrt{\kappa_{e} / \Gamma^{44}}$ ), to fully exploit the $T_{e}$ profile with expected maximum at $L / 2^{26,29}$, i.e. the WG centre.

Electrical characterisation. An image of the full GPD after fabrication, obtained by SEM, is in Fig. 4a, revealing a well-aligned WG, LMH, and split-gate structure. We first verify gate tunability of the SLG channel by measuring the drain-source current $\left(I_{\mathrm{DS}}\right)$ at a fixed drain-source voltage $\left(V_{\mathrm{DS}}\right)$ while varying the two gatevoltages. The resulting resistance map (Fig. 4b) shows a cross pattern, which confirms that four junction constellations ( $p-n$, $n-p, n-n, p-p)$ can be generated in the channel ${ }^{26}$. In order to extract the contact resistance, $R_{\text {contact, }}$ and $\mu$ from the GPD directly (rather than from a four-probe reference structure made from a second $\mathrm{LMH}$ ), then used to estimate $S$, we utilize the measured transfer curve at homogenous channel doping in Fig. 4c and plot the device resistance as function of the inverse carrier concentration $(1 / n)$ for electron (Fig. 4e) and hole (Fig. 4f) doping. By fitting the linear part (as $1 / n \rightarrow 0$ ) of these plots, as for ref. ${ }^{73}, R_{\text {contact }}$ can be obtained from the intersection of the fit curve and y-axis, while the residual carrier concentration, $n_{0}$, is found from the intersection between the fit curve and a horizontal line through the maximum. Using these values, we then model the total device resistance as for ref. ${ }^{26}: R_{\text {total }}=R_{\text {contact }}+\frac{L}{W} \frac{1}{e \mu n}$, with $n=\sqrt{n_{0}^{2}+\left[C_{\mathrm{ox}} / e\left(V_{\mathrm{G}}-V_{\mathrm{CNP}}\right)\right]^{2}}$, where $R_{\text {contact }}$ includes the contacts and the contribution from the ungated region, $V_{\mathrm{CNP}}$ is the gate voltage corresponding to the charge neutrality point (CNP, $\left.E_{\mathrm{F}}=0 \mathrm{meV}\right), C_{\mathrm{ox}}$ is the gate capacitance, and $\mu$ is used as a fit parameter. The original data (solid line) and the model (dashed line) are compared in Fig. $4 \mathrm{c}$. We get $R_{\text {contact }} \sim 400 \Omega$ and $\sim 530 \Omega$, as well as $\mu_{\mathrm{e}} \sim 17,700 \mathrm{~cm}^{2} / \mathrm{Vs}$ and $\mu_{\mathrm{h}} \sim 11,800 \mathrm{~cm}^{2} / \mathrm{Vs}$ for electrons (red lines) and holes (blue lines), respectively. This demonstrates a PIC-integrated, LMH-based PD with high $\left(>10^{4} \mathrm{~cm}^{2} / \mathrm{Vs}\right) \mu$, while refs. ${ }^{25,74}$ used $\mathrm{hBN}$ encapsulation for alternative detection concepts.

Steady-state photoresponse. For optical characterisation, we first couple modulated light (ON-OFF) with a duty cycle of $50 \%$ from a tuneable laser source (Agilent 81680A) into the bus WG using an optical single-mode fibre. While varying the potential at the two gate electrodes $\left(V_{\mathrm{G} 1}, V_{\mathrm{G} 2}\right)$, the photoresponse of the unbiased GPD ( $V_{\mathrm{DS}}=0 \mathrm{mV}$, to avoid dark currents impairing the noise performance) is recorded using a lock-in amplifier. Since the PTE effect as electromotive force is intrinsically best read-out as open circuit potential difference ${ }^{4,75}$, we record the response as $V_{\mathrm{PTE}}$, rather than measuring the resulting short-circuit photocurrent, which depends on external factors, such as $R_{\text {contact. Figure }} 4 \mathrm{~g}$ shows a photoresponsivity map measured on resonance at $1555.87 \mathrm{~nm}$, from which we extract a maximum $R_{[\mathrm{V} / \mathrm{W}]} \sim 90 \mathrm{~V} / \mathrm{W}$. The six-fold 
a

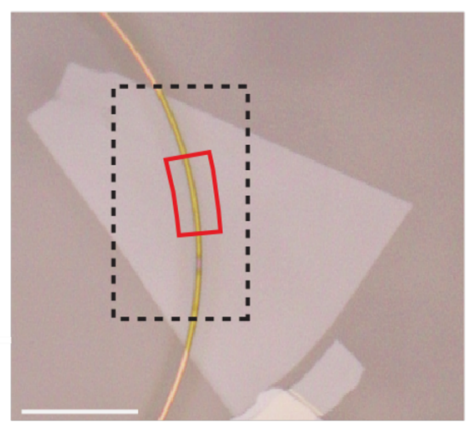

C

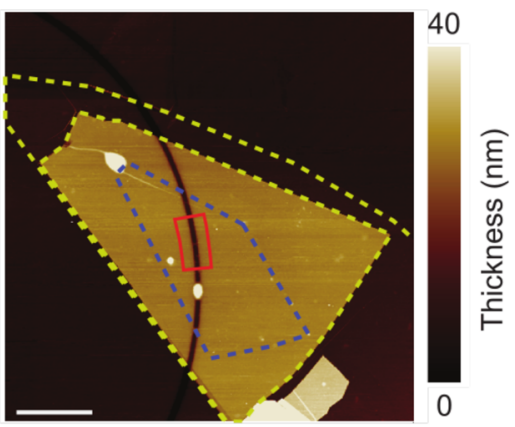

b

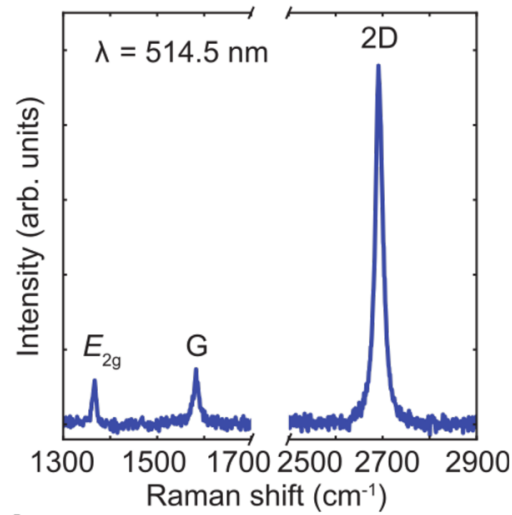

d

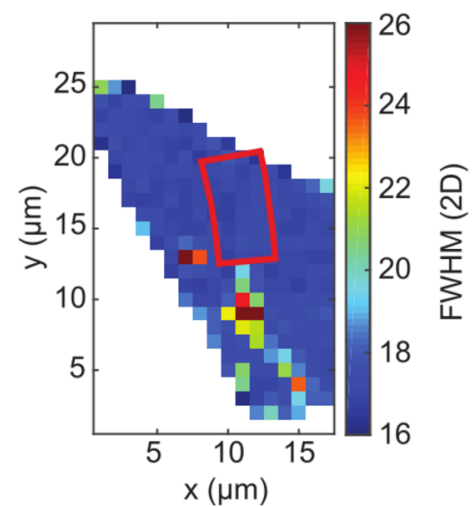

Fig. 3 LMH characterisation. a Microscope image of hBN/SLG/hBN on ring resonator. The black dashed line indicates the area over which the Raman map in $\mathbf{d}$ is measured. Scale bar, $10 \mu \mathrm{m}$. b Raman spectrum measured at the position of the final device. c AFM image of LMH. The yellow dashed line indicates the area of top and bottom hBN. The blue line indicates the SLG area. Scale bar, $10 \mu \mathrm{m}$. $\mathbf{d}$ Raman map of FWHM(2D). The red box marks the position of the final device in $\mathbf{a}, \mathbf{c}, \mathbf{d}$.

pattern, with the highest photoresponse for bipolar (p-n, n-p) junctions, and a sign-change across the diagonal $\left(V_{\mathrm{G} 1}=V_{\mathrm{G} 2}\right)$ for unipolar (n-n, p-p) junctions in the SLG channel, confirms that the PTE effect dominates the conversion of photons into electrical signal (rather than a photovoltaic conversion with a two-fold pattern in photovoltage over the same measurement range) ${ }^{43,44}$. Our $R_{\text {[V/W] }}$ outperforms the current state-of-the-art for waveguideintegrated PTE-GPDs, $R_{[\mathrm{V} / \mathrm{W}]} \sim 3-12 \mathrm{~V} / \mathrm{W}^{25,29,33,76}$, by around one order of magnitude.

High-speed photoresponse. To determine the BW, we modulate $\mathrm{CW}$ light at $1555.87 \mathrm{~nm}$ from the same source using a commercial (Thorlabs LN05S-FC) intensity modulator (lithium niobate, $f_{3 \mathrm{~dB}}=40 \mathrm{GHz}$ ) and couple it into the device. While tuning the modulation frequency of the external modulator, we monitor the GPD response with an electrical spectrum analyzer (Agilent PSX N9030A), while the gate bias $\left(V_{\mathrm{G} 1}=-0.5 \mathrm{~V}, V_{\mathrm{G} 2}=-2.1 \mathrm{~V}\right)$ is set at an operating point where $R_{[\mathrm{V} / \mathrm{W}]}$ is largest. This gives a 3 - $\mathrm{dB}$ bandwidth $\sim 12 \mathrm{GHz}$, Fig. $4 \mathrm{~h}$, as expected from the design of the passive photonic structure and the cavity-imposed limit calculated via Eq. (3).

Power and wavelength dependence. Figures $5 \mathrm{a}, \mathrm{b}$ plot the wavelength dependence of optical transmission and photovoltage for $V_{\mathrm{G} 1}=1 \mathrm{~V}, V_{\mathrm{G} 2}=-1 \mathrm{~V}$ and various $P_{\mathrm{in}}$. The maxima of the $V_{\mathrm{PTE}}$ traces match the resonance minima in $P_{\text {trans }}$, confirming the proportionality between the two, as SLG dominates absorption for our resonator-based PDs. This is also shown in Supplementary Note 2 and Supplementary Fig. 2, where we plot the estimated absorption and resulting $V_{\mathrm{PTE}}$ at low $(<0.1 \mathrm{~mW}) P_{\text {in }}$ on a linear scale. The shift of the resonance and its asymmetry for higher $P_{\text {in }}(>0.2 \mathrm{~mW})$ are attributed to the power-dependent change of the effective refractive index of the Si WG through a thermo-optic effect ${ }^{52,77}$. Extracting the power-dependent maxima in $V_{\mathrm{PTE}}$ allows us to estimate $T_{e}$ at resonance, and plot the $R_{[\mathrm{V} / \mathrm{W}]}$ power dependence.

The junction carrier temperature, $T_{e, j}$, can be written as (see Methods):

$$
T_{\mathrm{e}, \mathrm{j}}=\sqrt{2\left|\frac{V_{\mathrm{PTE}}}{\zeta_{1}-\zeta_{2}}\right|+T_{0}^{2}}
$$

where $T_{0}=294 \mathrm{~K}$ is the room temperature and $\zeta_{1,2}=\frac{\pi^{2} k_{\mathrm{B}}^{2}}{3 e \sigma} \frac{\mathrm{d} \sigma}{\mathrm{d} \epsilon}$ at $\epsilon=E_{F}$, in analogy to Eq. (2). In order to estimate $\zeta_{1,2}$, following the same method used to determine $S$ in Fig. $4 \mathrm{~d}$, we use $R_{\text {contact }}$ and $\mu$ as obtained from the electrical measurements at homogeneous channel doping. The resulting $T_{e}$ extracted for different $P_{\text {in }}$ is in Fig. 5c. The carriers reach $T_{e} \sim 400 \mathrm{~K}$ for $P_{\text {in }} \sim$ $0.6 \mathrm{~mW}$, due to cavity-enhanced light-matter interaction. The $T_{e}$ power dependence can be fitted by the heat equation 44,70 , neglecting diffusive cooling through the contacts (see Methods):

$$
T_{\mathrm{e}, \mathrm{j}}=\left(\beta P_{\mathrm{IN}}+T_{0}^{\delta}\right)^{\frac{1}{\delta}}
$$

with $\delta \sim 3^{78}$ and $\beta$ a fitting parameter.

The associated, power-dependent, $R_{[\mathrm{V} / \mathrm{W}]}$ is in Fig. $5 \mathrm{~d}$. For small $P_{\text {in }}(<0.2 \mathrm{~mW})$, we get a constant $R_{[\mathrm{V} / \mathrm{W}]}$. For $P_{\text {in }}>0.2 \mathrm{~mW}$ we have sublinear scaling between $V_{\mathrm{PTE}}$ and $P_{\text {in }}$, giving a $R_{[\mathrm{V} / \mathrm{W}]}$ 
a

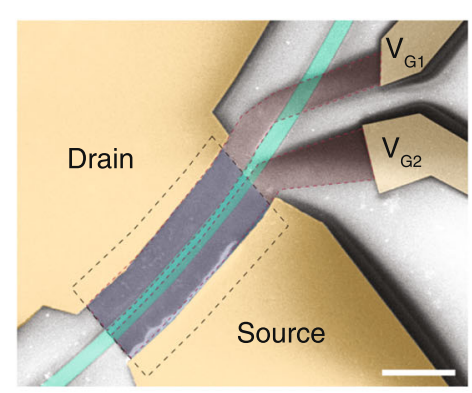

C

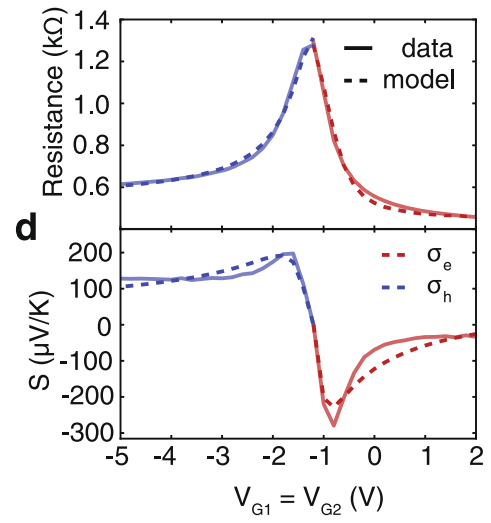

g

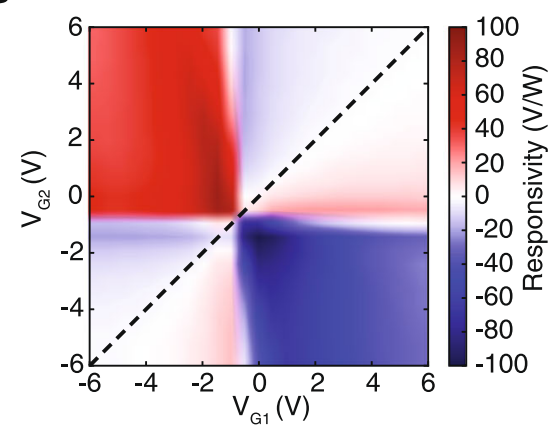

e b
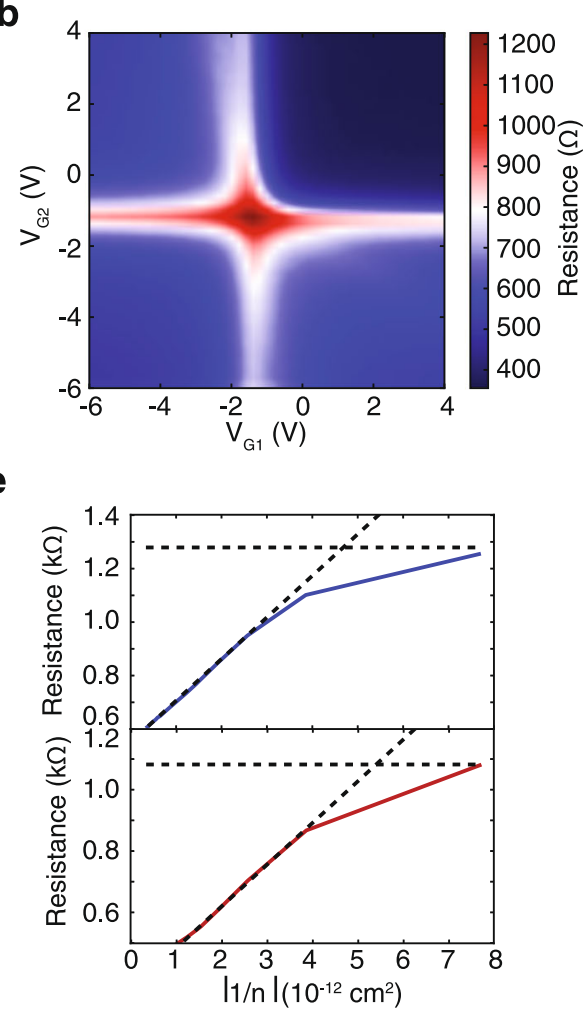

h

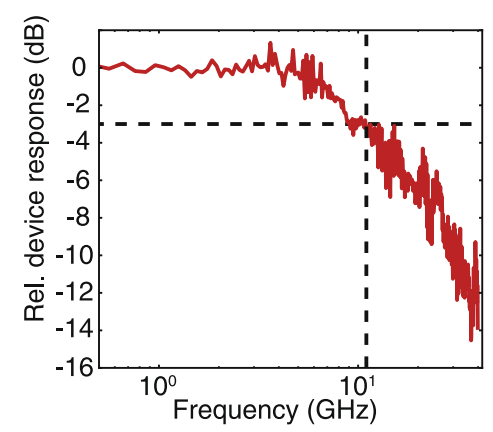

Fig. 4 GPD characterisation. a False-colour SEM image of our GPD, showing Cr/Au contacts (yellow), Si WG (green), contacted hBN/SLG/hBN (blue, dashed line under contacts), and SLG gates (red). Scale bar: $2 \mu \mathrm{m}$. b Resistance map demonstrating independent tunability of charge carrier concentration in the SLG channel via $V_{\mathrm{G} 1}$ and $V_{\mathrm{G} 2}$. $\mathbf{c}$ Electrical characterization at homogeneous channel doping (solid lines, measured data; dashed lines, model). d Calculated $S$ based on the electrical data in $\mathbf{c}$. e, $\mathbf{f}$ ) Resistance vs. inverse carrier concentration for $\mathbf{e}$ electron and $\mathbf{f}$ hole doping. $\mathbf{g}$ Photoresponse at zero bias on resonance $(\lambda=1555.87 \mathrm{~nm})$. h Frequency response. The $3-\mathrm{dB}$ cutoff frequency, marked by intersecting dashed lines, is $\sim 12 \mathrm{GHz}$.

\section{a}

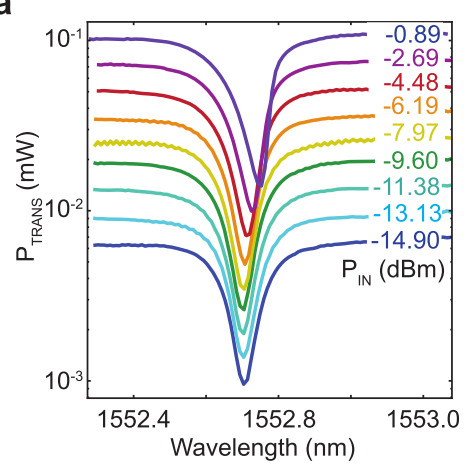

b

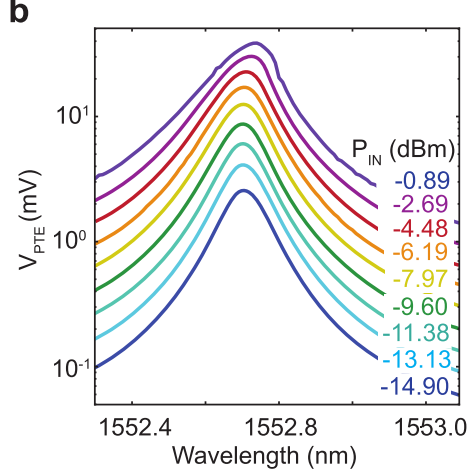

C

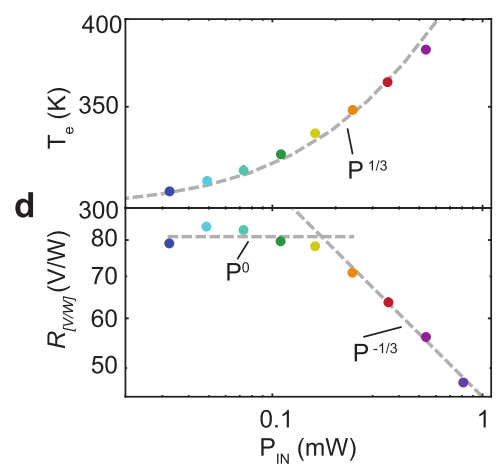

Fig. 5 Wavelength and power dependence. a Transmitted power for various $P_{\mathrm{in}}$. $\mathbf{b}$ Photovoltage for a fixed gate voltage combination $\left(V_{\mathrm{G} 1}=1 \mathrm{~V}, V_{\mathrm{G} 2}=-1 \mathrm{~V}\right)$ for various input powers (same color code as for the transmission in a). c $T_{e}$ calculated from the photoresponse in $\mathbf{b}$ and $S$ in Fig. $4 \mathrm{~d}$. d Power-dependent $R_{[V / W]}$. 
drop according to $R_{[\mathrm{V} / \mathrm{w}]} \propto P_{\mathrm{in}}^{-1 / 3}$ due to the $T_{e}$ dependence of the electronic heat capacity ${ }^{78,79}$.

\section{Discussion}

In order to assess our GPD performance against non-SLG-based ones, where typically a photocurrent $I_{\mathrm{ph}}$ is generated ${ }^{14}$, we compare two representative optical receiver implementations: a conventional (i.e. non-SLG) system based on a wafer-scale, commercial, high-speed $(>50 \mathrm{GHz})$ Ge photodiode with $R_{[\mathrm{A} / \mathrm{W}]} \sim$ $0.5 \mathrm{~A} / \mathrm{W}^{80}$, and a receiver based on our GPD. In both systems the same amplifier is employed to obtain a $>200 \mathrm{mV}$ output voltage swing $\left(V_{\text {OUT }}\right)$, as required for driving the subsequent clock and data recovery CMOS circuit. For simplicity we assume that the amplifier represents a capacitive load.

Our GPDs are based on the PTE effect, where an electromotive force directly provides a voltage, rather than a current ${ }^{8,79}$. In case of $\mathrm{Ge}$, an additional TIA is needed to convert the photocurrent into a voltage for further signal processing ${ }^{81}$. In the TIA we consider a feedback resistor $R_{\mathrm{F}}=(90 \mathrm{~V} / \mathrm{W}) /(0.5 \mathrm{~A} / \mathrm{W})=180 \Omega$, which assures the same $V_{\text {OUT }}$ for same optical input power in both cases. Neglecting any noise other than thermal noise produced by $R_{\mathrm{F}}$, we estimate for the conventional receiver a lower limit for the sensitivity $\bar{P}_{\text {sens }}=Q i_{\mathrm{n}} / R_{[\mathrm{A} / \mathrm{W}]}=12.6 \mu \mathrm{W} \sim-19 \mathrm{dBm}$ at a bit-error-rate (i.e. probability of false identification of a bit by the receiver decision circuit ${ }^{82}$ ) $\mathrm{BER}=10^{-9}$. We calculate the thermal noise current as $i_{\mathrm{n}}=\sqrt{4 k_{\mathrm{B}} \cdot T \cdot B W / R_{\mathrm{F}}}$ with $\mathrm{BW}=12 \mathrm{GHz}$, as in our GPDs, and a $Q$ factor (i.e. required signal-to-noise ratio to get a specific $\left.\mathrm{BER}^{83}\right) \sim 6$ from $^{82} \mathrm{BER}=\frac{1}{2} \operatorname{erfc}(Q / \sqrt{2})$. For our GPDs, we estimate $\bar{P}_{\text {sens }}=Q v_{\mathrm{n}} / R_{[\mathrm{V} / \mathrm{W}]} \sim-16 \mathrm{dBm}$ for same BER and $\mathrm{BW}$, where $v_{\mathrm{n}}=\sqrt{4 k_{\mathrm{B}} \cdot T \cdot B W \cdot R_{\mathrm{G}}}$, and $R_{\mathrm{G}} \sim 800 \Omega$ is the total device resistance. Thus, in contrast to previous reports of both PTE $^{25-27,29,33,76}$ and non-PTE ${ }^{30-32}$ GPDs for integrated photonics, $\bar{P}_{\text {sens }}$ of our GPD-based receiver is on par with mature semiconductor technology, and could be further improved by reducing $R_{\text {contact }}$, which dominates the total device resistance, thus being the primary source of thermal noise. The natural generation of a voltage makes the need for a TIA obsolete, with a reduction of energy-per-bit cost and system foot-print.

In summary, we reported photo-thermoelectric GPDs, integrated on Si microring resonators. By tuning the SLG coverage on top of the resonator, we optimised the round-trip propagation losses inside the cavity to get critical coupling, achieving $>90 \%$ light absorption in $\sim 6 \mu \mathrm{m}$ SLG. The resulting carrier heating on such compact lengths enables high peak $T_{e} \sim 400 \mathrm{~K}$ in the GPDs. In combination with high $\left(>10^{4} \mathrm{~cm}^{2} / \mathrm{Vs}\right)$ mobility, obtained by encapsulating the SLG channel in hBN, this allowed us to get $R_{[\mathrm{V} / \mathrm{W}]} \sim 90 \mathrm{~V} / \mathrm{W}$. Our bias-free, Johnson-noise limited GPDs, with voltage output, are a more power-efficient alternative to state of the art commercial PDs used in optical interconnects. Hotcarrier effects in SLG can be exploited for receiver architectures where current-to-voltage conversion, traditionally performed by transimpedance amplifiers, can be bypassed for a reduction in energy-per-bit cost and system foot-print.

\footnotetext{
Methods

Sample fabrication. The LMH is made as follows ${ }^{55}$ : SLG and hBN flakes of different thicknesses $\left(t_{\text {bottom }} \sim 3 \mathrm{~nm}, t_{\text {top }} \sim 20 \mathrm{~nm}\right)$ are prepared on $\mathrm{Si} / \mathrm{SiO}_{2}\left(t_{\mathrm{SiO}_{2}}=\right.$ $285 \mathrm{~nm}$ ) by MC of bulk graphite (Graphenium) and hBN single crystals grown at high pressure and temperature as detailed in ref. ${ }^{84}$. The thickness of the bottom $\mathrm{hBN}, t_{\text {bottom }}$, is chosen with the following trade-off: sufficiently thin $(<5 \mathrm{~nm})$ to ensure $\alpha_{\text {SLG }}$ (thus $\xi$ ) comparable to the initial experiment used to find $W$, but sufficiently thick $(\sim \mathrm{nm})$ that high $\left(>10^{4} \mathrm{~cm}^{2} / \mathrm{Vs}\right) \mu$ is achieved, due to reduced (in comparison to $\mathrm{SiO}_{2}$ ) carrier inhomogeneities, roughness, and charge impurities $55,85,86$. A micro-manipulator and a PC/PDMS stamp are then used to pick up and stack the flakes at $50^{\circ} \mathrm{C}$ (Fig. 2a). In order to clean the $\mathrm{LMH}$ interfaces, the target photonic chip is then heated to $180^{\circ} \mathrm{C}^{55}$, while we align the $\mathrm{LMH}$ to the
} $\mathrm{Si}$ WG. We then laminate the PC film onto the target substrate, pushing contamination blisters, formed at the SLG/hBN interfaces, out of the GPD channel region and placing the $\mathrm{LMH}$ on the WG, Fig. $2 \mathrm{~b}$.

After the PC film is dissolved in chloroform, we perform EBL (Raith EBPG 5200) to define the GPD channel geometry via a PMMA etch mask. To transfer this pattern to the $\mathrm{LMH}$, we use two dry etching steps. First, to achieve etch selectivity between LMH and underlying photonic circuit, we use a reactive ion etcher (RIE, Plasma-Therm) with a forward radio frequency power $\sim 80 \mathrm{~W}$ and an $\mathrm{SF}_{6}$ flow $\sim 80 \mathrm{sccm}$. As reported by ref. ${ }^{87}$, these conditions allow fast $(>200 \mathrm{~nm} / \mathrm{min})$ etching of $\mathrm{hBN}$, slow $(<7 \mathrm{~nm} / \mathrm{min})$ etching of $\mathrm{SiO}_{2}$, while SLG is not etched, serving as etch stop on the bottom hBN flake (Fig. 2c). We then expose the $\mathrm{LMH}$ to low power (3W) $\mathrm{O}_{2}$ plasma to remove all excess SLG, leaving behind the fully shaped GPD channel (Fig. 2d). A second EBL step, electron beam evaporation $(5 \mathrm{~nm} \mathrm{Cr} / 50 \mathrm{~nm} \mathrm{Au})$, and lift-off in acetone are then used to contact the exposed SLG channel edges (Fig. 2e). To fabricate the split-gate structure on top of the $\mathrm{LMH}$, required to create a p-n juntion in the GPD channel, a transparent (at $\lambda \sim 1.55 \mu \mathrm{m}$ ) conductor that does not affect $\xi$ (thus $W$ ) is needed. A second layer of SLG, sufficiently high ( $\sim$ tens $\mathrm{nm}$ ) above the WG to leave $\alpha_{\text {SLG }}$ unaltered, can be used for this. We therefore thermally evaporate $1 \mathrm{~nm} \mathrm{Al}$ as seed layer on the top $\mathrm{hBN}$, and atomic layer deposit (ALD, Savannah) $t_{\mathrm{ox}} \sim 20 \mathrm{~nm} \mathrm{Al} \mathrm{O}_{3}$ as additional gate dielectric and spacer between channel and gate electrodes (Fig. 2f). The combined thickness of the top-gate dielectric $\left(t_{\text {top }}+t_{\text {ox }}\right)$ ensures both low ( $2 \mathrm{~V}$ or less) gate biases at the operating point of the GPD and, from eigenmode Lumerical simulations, a change in $\alpha_{\mathrm{SLG}}<25 \%$.

To ensure alignment between LMH and gate SLG, we transfer a continuous film of SLG, grown by chemical vapor deposition (CVD) on $\mathrm{Cu}$, following the process described in ref. ${ }^{88}$, using a PMMA support membrane ${ }^{89}$ on the SLG/Cu substrate. We etch $\mathrm{Cu}$ in ammonium persulfate, transfer the PMMA/SLG stack onto the photonic chip (Fig. 2g), and remove the PMMA by immersion in acetone. We then use two additional EBL steps, $\mathrm{O}_{2}$ plasma etching, and electron beam evaporation, to define the SLG split-gate geometry (Fig. 2h) and fabricate metal contacts to these gates (Fig. 2i). Finally, we perform optical lithography on a laser writer (MicroTECH LW405) and wet etching in HF to get access to drain and source contact pads.

Temperature estimation. The junction $T_{e}$ can be extracted from powerdependent photovoltage measurements. Rewriting Eq. (2) as $S=\zeta T_{\mathrm{e}}$, where $\zeta=$ $-\left(\pi^{2} k_{\mathrm{B}}^{2}\right) / 3 e \sigma \mathrm{d} \sigma / \mathrm{d} \epsilon$ at $\epsilon=E_{F}$, and using this in Eq. (1), we get $V_{\mathrm{PTE}}=\int S(x) \nabla T_{\mathrm{e}}(x)$ $\mathrm{d} x=\int \zeta(x) T_{\mathrm{e}}(x) \nabla T_{\mathrm{e}}(x) \mathrm{d} x$. Integration over the $\mathrm{p}$ - $\mathrm{n}$-junction gives $V_{\text {PTE }}=\zeta_{1} \int_{-\frac{L}{2}}^{0} T_{\mathrm{e}}(x) \frac{\mathrm{d} T_{\mathrm{e}}}{\mathrm{d} x} \mathrm{~d} x+\zeta_{2} \int_{0}^{\frac{L}{2}} T_{\mathrm{e}}(x) \frac{\mathrm{d} T_{\mathrm{e}}}{\mathrm{d} x} \mathrm{~d} x=\frac{\zeta_{1}-\zeta_{2}}{2}\left(T_{\mathrm{e}, \mathrm{j}}^{2}-T_{0}^{2}\right)$. From this, we extract the junction $T_{e}$ from the measured photovoltage as $T_{\mathrm{e}, \mathrm{j}}=\sqrt{2\left|\frac{V_{\mathrm{PTE}}}{\zeta_{1}-\zeta_{2}}\right|+T_{0}^{2}}$. In the most general case, the $T_{e}$ profile can be calculated from the heat equation ${ }^{44,70}$, including diffusive cooling through contacts and cooling through the phonon bath: $\frac{\mathrm{d} q}{\mathrm{~d} x}=-\frac{\mathrm{d}}{\mathrm{d} x}\left(\kappa_{\mathrm{e}} \frac{\mathrm{d}}{\mathrm{d} x} T_{\mathrm{e}}\right)+\frac{\kappa_{\mathrm{e}}}{L_{\text {coling }}^{2}}\left(T_{\mathrm{e}}^{\delta}-T_{0}^{\delta}\right)$, where $\frac{\mathrm{d} q}{\mathrm{~d} x}$ describes the heating of the system. The electronic thermal conductivity is given by $\kappa_{\mathrm{e}}=\sigma \mathcal{L}_{0} T_{\mathrm{e}}{ }^{90}$, with Lorenz number $\mathcal{L}_{0}$, while a $T_{e}^{\delta}$ dependence with $\delta \sim 3$ is characteristic for $\mathrm{SLG}^{70,91}$. Assuming the distance from the heat source to the heat sink larger than the cooling length $\left(L_{\text {channel }}>2 L_{\text {cooling }}\right)$, the heat equation becomes $\frac{\mathrm{d} q}{\mathrm{~d} x}=\frac{\kappa_{\mathrm{e}}}{L_{\text {cooling }}^{2}}\left(T_{\mathrm{e}}^{\delta}-T_{0}^{\delta}\right)$, with $\frac{\mathrm{d} q}{\mathrm{~d} x}=\alpha P_{\text {in }} / A, \alpha P_{\text {in }}$ the fraction of the absorbed power, and $A$ the heated area. We thus get $T_{\mathrm{e}, \mathrm{j}}=\left(\beta P_{\mathrm{in}}+T_{0}^{\delta}\right)^{\frac{1}{\delta}}$, with $\beta$ a fit parameter.

\section{Data availability}

Data supporting the findings of this study are available from the corresponding authors upon reasonable request.

Received: 5 July 2020; Accepted: 14 April 2021; Published online: 18 June 2021

\section{References}

1. Atabaki, A. H. et al. Integrating photonics with silicon nanoelectronics for the next generation of systems on a chip. Nature 556, 349-354 (2018).

2. Cheng, Q., Bahadori, M., Glick, M., Rumley, S. \& Bergman, K. Recent advances in optical technologies for data centers: a review. Optica $\mathbf{5}$ 1354-1370 (2018)

3. Subbaraman, H. et al. Recent advances in silicon-based passive and active optical interconnects. Opt. Express 23, 2487-2511 (2015).

4. Romagnoli, M. et al. Graphene-based integrated photonics for next-generation datacom and telecom. Nat. Rev. Mater. 3, 392-414 (2018).

5. Bonaccorso, F., Sun, Z., Hasan, T. \& Ferrari, A. C. Graphene photonics and optoelectronics. Nat. Photonics 4, 611-622 (2010).

6. Urich, A., Unterrainer, K. \& Mueller, T. Intrinsic response time of graphene photodetectors. Nano Lett. 11, 2804-2808 (2011).

7. Xia, F., Mueller, T., Lin, Y., Valdes-Garcia, A. \& Avouris, P. Ultrafast graphene photodetector. Nat. Nanotechnol. 4, 839-843 (2009). 
8. Koppens, F. H. et al. Photodetectors based on graphene, other two-dimensional materials and hybrid systems. Nat. Nanotechnol. 9, 780-793 (2014).

9. Cai, X. et al. Sensitive room-temperature terahertz detection via the photothermoelectric effect in graphene. Nat. Nanotechnol. 9, 814-819 (2014).

10. Vivien, L. et al. Zero-bias 40Gbit/s germanium waveguide photodetector on silicon. Opt. Express 20, 1096-1101 (2012).

11. Salamin, Y. et al. $100 \mathrm{GHz}$ plasmonic photodetector. ACS Photonics $\mathbf{5}$, 3291-3297 (2018).

12. Michel, J., Liu, J. \& Kimerling, L. C. High-performance Ge-on-Si photodetectors. Nat. Photonics 4, 527-534 (2010).

13. Thomson, D. et al. Roadmap on silicon photonics. J. Opt. 18, 073003 (2016).

14. Chrostowski, L. \& Hochberg, M. Silicon Photonics Design: From Devices to Systems (Cambridge University Press, 2015).

15. Arafin, S. \& Coldren, L. A. Advanced InP photonic integrated circuits for communication and sensing. IEEE J. Sel. Top. Quantum Electron. 24, 1-12 (2018).

16. Nagarajan, R. et al. InP photonic integrated circuits. IEEE J. Sel. Top. Quantum Electron. 16, 1113-1125 (2010).

17. Liu, M. et al. A graphene-based broadband optical modulator. Nature $\mathbf{4 7 4}$, 64-67 (2011)

18. Phare, C. T., Daniel Lee, Y., Cardenas, J. \& Lipson, M. Graphene electro-optic modulator with $30 \mathrm{GHz}$ bandwidth. Nat. Photonics 9, 511-514 (2015).

19. Giambra, M. A. et al. High-speed double layer graphene electro-absorption modulator on SOI waveguide. Opt. Express 27, 20145-20155 (2019).

20. Sorianello, V. et al. Graphene-silicon phase modulators with gigahertz bandwidth. Nat. Photonics 12, 40-44 (2018).

21. Cassese, T. et al. Capacitive actuation and switching of add-drop graphenesilicon micro-ring filters. Photonics Res. 5, 762-766 (2017).

22. Pospischil, A. et al. CMOS-compatible graphene photodetector covering all optical communication bands. Nat. Photonics 7, 892-896 (2013).

23. Gan, X. et al. Chip-integrated ultrafast graphene photodetector with high responsivity. Nat. Photonics 7, 883-887 (2013).

24. Wang, X., Cheng, Z., Xu, K., Tsang, H. K. \& Xu, J. High-responsivity graphene/silicon-heterostructure waveguide photodetectors. Nat. Photonics 7 , 888-891 (2013).

25. Shiue, R. J. et al. High-responsivity graphene-boron nitride photodetector and autocorrelator in a silicon photonic integrated circuit. Nano Lett. 15, 7288-7293 (2015).

26. Schuler, S. et al. Controlled generation of a $\mathrm{p}-\mathrm{n}$ junction in a waveguide integrated graphene photodetector. Nano Lett. 16, 7107-7112 (2016).

27. Schuler, S. et al. Graphene photodetector integrated on a photonic crystal defect waveguide. ACS Photonics 5, 4758-4763 (2018).

28. Goykhman, I. et al. On-chip integrated, silicon-graphene plasmonic schottky photodetector with high responsivity and avalanche photogain. Nano Lett. 16, 3005-3013 (2016).

29. Muench, J. E. et al. Waveguide-integrated, plasmonic enhanced graphene photodetectors. Nano Lett. 19, 7632-7644 (2019).

30. Schall, D. et al. In Optical Fiber Communication Conference, M2I.4 (Optical Society of America, 2018).

31. Ma, P. et al. Plasmonically enhanced graphene photodetector featuring $100 \mathrm{Gbit} / \mathrm{s}$ data reception, high responsivity, and compact size. ACS Photonics 6, 154-161 (2019).

32. Ding, Y. et al. Ultra-compact integrated graphene plasmonic photodetector with bandwidth above $110 \mathrm{GHz}$. Nanophotonics 9, 317-325 (2020).

33. Mišeikis, V. et al. Ultrafast, zero-bias, graphene photodetectors with polymeric gate dielectric on passive photonic waveguides. ACS Nano 14, 11190-11204 (2020).

34. Giambra, M. A. et al. Wafer-scale integration of graphene-based photonic devices. ACS Nano 15, 3171-3187 (2021).

35. Gao, Y., Zhou, G., Zhao, N., Tsang, H. K. \& Shu, C. High-performance chemical vapor deposited graphene-on-silicon nitride waveguide photodetectors. Opt. Lett. 43, 1399-1402 (2018).

36. Cheng, Z., Wang, J., Xu, K., Tsang, H. K. \& Shu, C., In CLEO: Science and Innovations, STh1I-5 (Optical Society of America, 2015).

37. Wang, J. et al. Design of graphene-on-germanium waveguide electro-optic modulators at the $2 \mu \mathrm{m}$ wavelength. OSA Contin. 2, 749-758 (2019).

38. Kim, J. T. \& Choi, C. Graphene-based polymer waveguide polarizer. Opt. Express 20, 3556-3562 (2012).

39. Kleinert, M. et al. Graphene-based electro-absorption modulator integrated in a passive polymer waveguide platform. Optical Mater. Express 6, 1800-1807 (2016).

40. Qu, Z. et al. In Silicon Photonics XIII, 105371N, (International Society for Optics and Photonics, 2018).

41. Nair, R. R. et al. Fine structure constant defines visual transparency of graphene. Science 320, 1308-1308 (2008).

42. Youngblood, N. \& Li, M. Integration of $2 \mathrm{D}$ materials on a silicon photonics platform for optoelectronics applications. Nanophotonics 6, 1205-1218 (2017).

43. Gabor, N. M. et al. Hot carrier-assisted intrinsic photoresponse in graphene. Science 334, 648-652 (2011).
44. Song, J. C. W., Rudner, M. S., Marcus, C. M. \& Levitov, L. S. Hot carrier transport and photocurrent response in graphene. Nano Lett. 11, 4688-4692 (2011).

45. Xu, X., Gabor, N. M., Alden, J. S., van der Zande, A. M. \& McEuen, P. L. Photo-thermoelectric effect at a graphene interface junction. Nano Lett. 10, 562-566 (2010).

46. Brida, D. et al. Ultrafast collinear scattering and carrier multiplication in graphene. Nat. Commun. 4, 1987 (2013).

47. Tomadin, A., Brida, D., Cerullo, G., Ferrari, A. C. \& Polini, M. Nonequilibrium dynamics of photoexcited electrons in graphene: Collinear scattering, Auger processes, and the impact of screening. Phys. Rev. B 88, 035430 (2013)

48. Soavi, G. et al. Broadband, electrically tunable third-harmonic generation in graphene. Nat. Nanotechnol. 13, 583-588 (2018).

49. Echtermeyer, T. J. et al. Strong plasmonic enhancement of photovoltage in graphene. Nat. Commun. 2, 455-458 (2011).

50. Engel, M. et al. Light-matter interaction in a microcavity-controlled graphene transistor. Nat. Commun. 3, 906 (2012).

51. Furchi, M. et al. Microcavity-integrated graphene photodetector. Nano Lett. 12, 2773-2777 (2012).

52. Bogaerts, W. et al. Silicon microring resonators. Laser Photonics Rev. 6, 47-73 (2012).

53. Fang, Y., Armin, A., Meredith, P. \& Huang, J. Accurate characterization of next-generation thin-film photodetectors. Nat. Photonics 13, 1-4 (2019).

54. Balandin, A. A. Low-frequency $1 / \mathrm{f}$ noise in graphene devices. Nat. Nanotechnol. 8, 549-555 (2013).

55. Purdie, D. G. et al. Cleaning interfaces in layered materials heterostructures Nat. Commun. 9, 5387 (2018)

56. Rabus, D. G. Integrated ring resonators (Springer, 2007).

57. Yariv, A. Critical coupling and its control in optical waveguide-ring resonator systems. IEEE Photonics Technol. Lett. 14, 483-485 (2002).

58. Xu, Q., Schmidt, B., Shakya, J. \& Lipson, M. Cascaded silicon micro-ring modulators for WDM optical interconnection. Opt. Express 14, 9431-9436 (2006).

59. Zheng, X. et al. A tunable $1 \times 4$ silicon CMOS photonic wavelength multiplexer/ demultiplexer for dense optical interconnects. Opt. Express 18, 5151-5160 (2010).

60. Paschotta, R. Encyclopedia of laser physics and technology (Wiley-VCH, 2008).

61. Timurdogan, E. et al. An ultralow power athermal silicon modulator. Nat. Commun. 5, 4008 (2014).

62. Reed, G. T. \& Knights, A. P. Silicon Photonics: An Introduction (John Wiley \& Sons, 2004).

63. Novoselov, K. S. et al. Two-dimensional atomic crystals. Proc. Natl Acad. Sci. USA 102, 10451-10453 (2005).

64. Gan, X. et al. Strong enhancement of light-matter interaction in graphene coupled to a photonic crystal nanocavity. Nano Lett. 12, 5626-5631 (2012).

65. Reich, S. et al. Resonant Raman scattering in cubic and hexagonal boron nitride. Phys. Rev. B 71, 12 (2005).

66. Nemanich, R. J., Solin, S. A. \& Martin, R. M. Light scattering study of boron nitride microcrystals. Phys. Rev. B 23, 0 (1981).

67. Das, A. et al. Monitoring dopants by Raman scattering in an electrochemically top-gated graphene transistor. Nat. Nanotechnol. 3, 210-215 (2008).

68. Basko, D. M., Piscanec, S. \& Ferrari, A. C. Electron-electron interactions and doping dependence of the two-phonon Raman intensity in graphene. Phys. Rev. B 80, 165413 (2009).

69. Ferrari, A. C. \& Basko, D. M. Raman spectroscopy as a versatile tool for studying the properties of graphene. Nat. Nanotechnol. 8, 235-246 (2013).

70. Ma, Q. et al. Competing channels for hot-electron cooling in graphene. Phys. Rev. Lett. 112, 247401 (2014)

71. Freitag, M., Low, T. \& Avouris, P. Increased responsivity of suspended graphene photodetectors. Nano Lett. 13, 1644-1648 (2013).

72. Tielrooij, K. et al. Out-of-plane heat transfer in van der Waals stacks through electron-hyperbolic phonon coupling. Nat. Nanotechnol. 13, 41 (2018).

73. Zhang, Z., Xu, H., Zhong, H. \& Peng, L. Direct extraction of carrier mobility in graphene field-effect transistor using current-voltage and capacitance-voltage measurements. Appl. Phys. Lett. 101, 213103 (2012).

74. Bie, Y. et al. A MoTe2-based light-emitting diode and photodetector for silicon photonic integrated circuits. Nat. Nanotechnol. 12, 1124-1129 (2017).

75. Song, J. C. W. \& Levitov, L. S. Energy flows in graphene: hot carrier dynamics and cooling. J. Phys. Condens. Matter 27, 164201 (2015).

76. Marconi, S. et al. Photo thermal effect graphene detector featuring $105 \mathrm{Gbit}$ s-1 NRZ and 120 Gbit s-1 PAM4 direct detection. Nat. Commun. 12, 806 (2021).

77. Fainman, Y. et al. Silicon nanophotonic devices for chip-scale optical communication applications. Appl. Opt. 52, 613-624 (2013).

78. Graham, M. W., Shi, S., Ralph, D. C., Park, J. \& McEuen, P. L. Photocurrent measurements of supercollision cooling in graphene. Nat. Phys. 9, 103 (2013).

79. Tielrooij, K. et al. Generation of photovoltage in graphene on a femtosecond timescale through efficient carrier heating. Nat. Nanotechnol. 10, 437-443 (2015). 
80. Absil, P. P. et al. Silicon photonics integrated circuits: a manufacturing platform for high density, low power optical I/O's. Opt. Express 23, 9369-9378 (2015).

81. Okamoto, D. et al. A $25-\mathrm{Gb} / \mathrm{s} 5 \times 5 \mathrm{~mm} 2$ chip-scale silicon-photonic receiver integrated with 28-nm CMOS transimpedance amplifier. J. Lightwave Technol. 34, 2988-2995 (2016).

82. Agrawal, G. P. Fiber-optic communication systems (John Wiley \& Sons, 2012).

83. Bergano, N. S., Kerfoot, F. \& Davidsion, C. Margin measurements in optical amplifier system. IEEE Photonics Technol. Lett. 5, 304-306 (1993).

84. Taniguchi, T. \& Watanabe, K. Synthesis of high-purity boron nitride single crystals under high pressure by using Ba-BN solvent. J. Cryst. Growth $\mathbf{3 0 3}$, 525-529 (2007).

85. Dean, C. R. et al. Boron nitride substrates for high-quality graphene electronics. Nat. Nanotechnol. 5, 722 (2010).

86. Wang, L. et al. One-dimensional electrical contact to a two-dimensional material. Science 342, 614-617 (2013).

87. Jessen, B. S. et al. Lithographic band structure engineering of graphene. Nat. Nanotechnol. 14, 340 (2019).

88. $\mathrm{Li}, \mathrm{X}$. et al. Large-area synthesis of high-quality and uniform graphene films on copper foils. Science 324, 1312-1314 (2009)

89. Bonaccorso, F. et al. Production and processing of graphene and $2 \mathrm{~d}$ crystals. Mater. Today 15, 564-589 (2012).

90. Kittel, C. Introduction to solid state physics (Wiley New York, 1996).

91. Song, J. C. W., Reizer, M. Y. \& Levitov, L. S. Disorder-assisted electron-phonon scattering and cooling pathways in graphene. Phys. Rev. Lett. 109, 5 (2012).

\section{Acknowledgements}

We thank S. Wachter, D. Schall, A. Tomadin, J. Marcellino for useful discussions. We acknowledge funding from the EU Graphene Flagship, ERC grant Hetero2D, DSTL EPSRC grants EP/L016087/1, EP/K01711X/1, EP/K017144/1, EP/N010345/1.

\section{Author contributions}

S.S., J.E.M., A.C.F. and T.M. conceived the device. A.C.F. and T.M. supervised the work S.S. and J.E.M. fabricated the devices and performed the experiments. S.S., J.E.M., T.M. and A.C.F. analysed and discussed the data. J.E.M. and A.R. built the measurement setup.
O.B. prepared CVD SLG. T.T. and K.W. prepared hBN. D.V.T. and V.S. designed and provided the SOI waveguide substrates. S.S., J.E.M., T.M. and A.C.F. wrote the paper, with input from all authors.

\section{Competing interests}

The authors declare no competing interests.

\section{Additional information}

Supplementary information The online version contains supplementary material available at https://doi.org/10.1038/s41467-021-23436-x.

Correspondence and requests for materials should be addressed to A.C.F. or T.M

Peer review information Nature Communications thanks the anonymous reviewers for their contribution to the peer review of this work.

Reprints and permission information is available at http://www.nature.com/reprints

Publisher's note Springer Nature remains neutral with regard to jurisdictional claims in published maps and institutional affiliations.

(c) (i) Open Access This article is licensed under a Creative Commons Attribution 4.0 International License, which permits use, sharing, adaptation, distribution and reproduction in any medium or format, as long as you give appropriate credit to the original author(s) and the source, provide a link to the Creative Commons license, and indicate if changes were made. The images or other third party material in this article are included in the article's Creative Commons license, unless indicated otherwise in a credit line to the material. If material is not included in the article's Creative Commons license and your intended use is not permitted by statutory regulation or exceeds the permitted use, you will need to obtain permission directly from the copyright holder. To view a copy of this license, visit http://creativecommons.org/ licenses/by/4.0/.

(C) The Author(s) 2021 